\title{
Toward Modeling the Aromatic/aliphatic Separation by Extractive Distillation with Tricyanomethanide-based Ionic Liquids Using CPA EoS
}

Miguel Ayuso ${ }^{a}$, Pablo Navarro ${ }^{b, c, *}$, André M. Palma ${ }^{b}$, Marcos Larriba ${ }^{a, b}$, Noemí DelgadoMellado $^{a}$, Julián García ${ }^{a}$, Francisco Rodríguez $^{a}$, João A.P. Coutinho ${ }^{b}$, Pedro J. Carvalho ${ }^{b}$

${ }^{a}$ Department of Chemical Engineering, Complutense University of Madrid, Madrid, Spain.

${ }^{b}$ CICECO - Aveiro Institute of Materials, Department of Chemistry, University of Aveiro, Aveiro, Portugal.

${ }^{c}$ Department of Chemical Engineering, Autónoma University of Madrid, Madrid, Spain.

\section{Supporting Information}

\footnotetext{
* To whom correspondence should be addressed. E-mail address: pablo.navarro@uam.es (Pablo Navarro).
} 
Table of contents

Table S1. Vapor-liquid and Vapor-liquid-liquid Equilibria ${ }^{a}$ for Hydrocarbon

(1) $+\left[\mathrm{C}_{2} \mathrm{C}_{1} \mathrm{im}\right][\mathrm{TCM}](2)$

Table S2. Vapor-liquid and Vapor-liquid-liquid Equilibria ${ }^{a}$ for Hydrocarbon (1) $+\left[4-\mathrm{C}_{4} \mathrm{C}_{1} \mathrm{py}\right][\mathrm{TCM}](2)$

Table S3. Vapor-liquid and Vapor-liquid-liquid Equilibria ${ }^{a}$ for $n$-Hexane (1) +7 Benzene (2) $+\left[\mathrm{C}_{2} \mathrm{C}_{1}\right.$ im] $]$ TCM] (3) with $\mathrm{S} / \mathrm{F}=10$

Table S4. Vapor-liquid and Vapor-liquid-liquid Equilibria ${ }^{a}$ for $n$-Hexane (1) +9 Benzene (2) + [4- $\mathrm{C}_{4} \mathrm{C}_{1}$ py] $[\mathrm{TCM}]$ (3) with $\mathrm{S} / \mathrm{F}=10$

Table S5. Vapor-liquid and Vapor-liquid-liquid Equilibria ${ }^{a}$ for $n$-Octane (1) +11 $p$-Xylene (2) $+\left[\mathrm{C}_{2} \mathrm{C}_{1} \mathrm{im}\right][\mathrm{TCM}]$ (3) with $\mathrm{S} / \mathrm{F}=10$

Table S6. Vapor-liquid and Vapor-liquid-liquid Equilibria ${ }^{a}$ for $n$-Octane (1) $+\quad 13$ $p$-Xylene (2) $+\left[4-\mathrm{C}_{4} \mathrm{C}_{1}\right.$ py $][\mathrm{TCM}](3)$ with $\mathrm{S} / \mathrm{F}=10$ 
Table S1. Vapor-liquid and Vapor-liquid-liquid Equilibria ${ }^{a}$ for Hydrocarbon (1) + $\left[\mathrm{C}_{2} \mathrm{C}_{1}\right.$ im] [TCM] (2)

\begin{tabular}{|c|c|c|c|c|c|}
\hline$p / \mathbf{k P a}$ & $x_{1}$ & $p / \mathbf{k P a}$ & $x_{1}$ & $p / \mathbf{k P a}$ & $x_{1}$ \\
\hline \multicolumn{2}{|c|}{$\boldsymbol{T} / \mathbf{K}=323$} & \multicolumn{2}{|c|}{$\boldsymbol{T} / \mathbf{K}=363$} & \multicolumn{2}{|c|}{$\boldsymbol{T} / \mathbf{K}=403$} \\
\hline \multicolumn{6}{|c|}{ n-hexane $+\left[\mathrm{C}_{2} \mathrm{C}_{1}\right.$ im $][\mathrm{TCM}]$} \\
\hline 6.2 & 0.0017 & 7.8 & 0.0007 & 8.7 & 0.0005 \\
\hline 16.4 & 0.0067 & 22.2 & 0.0028 & 25.7 & 0.0017 \\
\hline 33.7 & 0.0158 & 51.4 & 0.0063 & 49.2 & 0.0025 \\
\hline 50.6 & 0.0273 & 83.3 & 0.0103 & 83.0 & 0.0053 \\
\hline 52.3 & 0.0596 & 111.4 & 0.0151 & 112.9 & 0.0067 \\
\hline 52.3 & 0.1624 & 168.2 & 0.0256 & 231.2 & 0.0168 \\
\hline 54.0 & 0.2048 & 183.8 & 0.0518 & 291.0 & 0.0220 \\
\hline \multirow[t]{7}{*}{54.3} & 1.0000 & 185.7 & 0.1485 & 345.3 & 0.0314 \\
\hline & & 188.3 & 0.2286 & 402.0 & 0.0430 \\
\hline & & 187.9 & 1.0000 & 450.2 & 0.0470 \\
\hline & & & & 492.2 & 0.0581 \\
\hline & & & & 492.2 & 0.1095 \\
\hline & & & & 493.1 & 0.2140 \\
\hline & & & & 492.9 & 1.0000 \\
\hline \multicolumn{6}{|c|}{$n$-octane $+\left[\mathrm{C}_{2} \mathrm{C}_{1} \mathrm{im}\right][\mathrm{TCM}]$} \\
\hline 4.4 & 0.0061 & 8.1 & 0.0097 & 11.4 & 0.0008 \\
\hline 5.9 & 0.0210 & 19.7 & 0.0239 & 26.9 & 0.0035 \\
\hline 6.2 & 0.0513 & 31.7 & 0.0495 & 50.2 & 0.0067 \\
\hline 6.3 & 0.0683 & 33.0 & 0.0790 & 75.5 & 0.0101 \\
\hline 6.4 & 0.0792 & 32.9 & 0.1079 & 95.0 & 0.0240 \\
\hline 6.6 & 0.1620 & 33.0 & 0.1747 & 105.8 & 0.0389 \\
\hline 6.7 & 0.2146 & 33.1 & 0.2294 & 113.2 & 0.0866 \\
\hline \multirow[t]{5}{*}{6.7} & 1.0000 & 33.4 & 1.0000 & 113.9 & 0.1830 \\
\hline & & & & 113.8 & 0.2418 \\
\hline & & & & 114.0 & 0.3014 \\
\hline & & & & 114.1 & 0.3570 \\
\hline & & & & 114.1 & 1.0000 \\
\hline
\end{tabular}

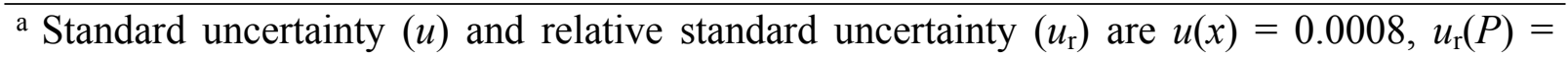
0.02 , and $u(T)=0.1 \mathrm{~K}$ 
Table S1. Continued

\begin{tabular}{|c|c|c|c|c|c|}
\hline$p / \mathbf{k P a}$ & $x_{1}$ & $p / \mathbf{k P a}$ & $x_{1}$ & $p / \mathbf{k P a}$ & $x_{1}$ \\
\hline \multicolumn{2}{|c|}{$\boldsymbol{T} / \mathbf{K}=323$} & \multicolumn{2}{|c|}{$\boldsymbol{T} / \mathbf{K}=363$} & \multicolumn{2}{|c|}{$\boldsymbol{T} / \mathbf{K}=403$} \\
\hline \multicolumn{6}{|c|}{ benzene $+\left[\mathrm{C}_{2} \mathrm{C}_{1}\right.$ im] $][\mathrm{TCM}]$} \\
\hline 0.9 & 0.0182 & 2.9 & 0.0172 & 6.3 & 0.0114 \\
\hline 2.1 & 0.0428 & 6.7 & 0.0393 & 16.6 & 0.0298 \\
\hline 4.1 & 0.0852 & 14.1 & 0.0782 & 34.4 & 0.0585 \\
\hline 6.2 & 0.1294 & 22.3 & 0.1153 & 55.4 & 0.0851 \\
\hline 8.0 & 0.1675 & 30.2 & 0.1507 & 75.3 & 0.1107 \\
\hline 12.0 & 0.2501 & 45.8 & 0.2271 & 116.0 & 0.1843 \\
\hline 15.9 & 0.3232 & 57.6 & 0.2951 & 159.8 & 0.2458 \\
\hline 18.9 & 0.3885 & 70.5 & 0.3576 & 184.9 & 0.2890 \\
\hline 21.8 & 0.4458 & 84.2 & 0.4129 & 227.8 & 0.3389 \\
\hline 24.2 & 0.4936 & 95.3 & 0.4717 & 254.8 & 0.3940 \\
\hline 27.4 & 0.5562 & 108.7 & 0.5234 & 290.5 & 0.4468 \\
\hline 30.7 & 0.6058 & 120.0 & 0.5747 & 312.8 & 0.5028 \\
\hline 33.1 & 0.6537 & 127.1 & 0.6242 & 343.4 & 0.5591 \\
\hline 35.2 & 0.6927 & 132.7 & 0.6700 & 360.3 & 0.6128 \\
\hline 35.5 & 0.7366 & 135.2 & 0.7172 & 377.3 & 0.6632 \\
\hline 35.9 & 0.7766 & 135.7 & 0.7590 & 378.1 & 0.7147 \\
\hline 36.0 & 0.8127 & 135.9 & 0.7994 & 378.4 & 0.7624 \\
\hline 36.1 & 1.0000 & 136.0 & 1.0000 & 378.4 & 1.0000 \\
\hline \multicolumn{6}{|c|}{$p$-xylene $+\left[\mathrm{C}_{2} \mathrm{C}_{1}\right.$ im $][\mathrm{TCM}]$} \\
\hline 0.2 & 0.0162 & 1.3 & 0.0137 & 4.2 & 0.0100 \\
\hline 0.5 & 0.0369 & 3.1 & 0.0336 & 8.7 & 0.0267 \\
\hline 1.0 & 0.0719 & 5.9 & 0.0666 & 17.8 & 0.0542 \\
\hline 1.5 & 0.1054 & 8.4 & 0.0976 & 24.9 & 0.0830 \\
\hline 1.9 & 0.1407 & 10.8 & 0.1310 & 30.2 & 0.1149 \\
\hline 2.7 & 0.2125 & 14.3 & 0.2010 & 44.8 & 0.1803 \\
\hline 3.3 & 0.2777 & 18.0 & 0.2575 & 57.9 & 0.2338 \\
\hline 3.8 & 0.3353 & 20.8 & 0.3208 & 70.3 & 0.2885 \\
\hline 4.3 & 0.3925 & 21.6 & 0.3799 & 79.5 & 0.3450 \\
\hline 4.3 & 0.4429 & 22.5 & 0.4321 & 80.8 & 0.4052 \\
\hline 4.3 & 0.4952 & 22.4 & 0.4896 & 80.8 & 0.4593 \\
\hline 4.2 & 0.5471 & 22.5 & 0.5391 & 81.1 & 0.5151 \\
\hline 4.2 & 0.5964 & 22.5 & 0.5898 & 80.6 & 0.5668 \\
\hline 4.3 & 0.6417 & 22.5 & 0.6395 & 80.7 & 0.6182 \\
\hline \multirow[t]{4}{*}{4.3} & 1.0000 & 22.6 & 1.0000 & 80.8 & 0.6650 \\
\hline & & & & 80.8 & 0.7123 \\
\hline & & & & 80.6 & 0.7624 \\
\hline & & & & 80.6 & 1.0000 \\
\hline
\end{tabular}

a Standard uncertainty $(u)$ and relative standard uncertainty $\left(u_{\mathrm{r}}\right)$ are $u(x)=0.0008, u_{\mathrm{r}}(P)=$ 0.02 , and $u(T)=0.1 \mathrm{~K}$ 
Table S2. Vapor-liquid and Vapor-liquid-liquid Equilibria ${ }^{a}$ for Hydrocarbon (1) + [4$\left.\mathrm{C}_{4} \mathrm{C}_{1} \mathrm{py}\right][\mathrm{TCM}](2)$

\begin{tabular}{|c|c|c|c|c|c|}
\hline$p / \mathbf{k P a}$ & $x_{1}$ & $p / \mathbf{k P a}$ & $x_{1}$ & $p / \mathbf{k P a}$ & $x_{1}$ \\
\hline \multicolumn{2}{|c|}{$\boldsymbol{T} / \mathbf{K}=323$} & \multicolumn{2}{|c|}{$\boldsymbol{T} / \mathbf{K}=363$} & \multicolumn{2}{|c|}{$T / \mathbf{K}=403$} \\
\hline \multicolumn{6}{|c|}{$n$-hexane $+\left[4-C_{4} C_{1} p y\right][T C M]$} \\
\hline 5.2 & 0.0028 & 6.7 & 0.0011 & 8.6 & 0.0002 \\
\hline 13.7 & 0.0151 & 19.5 & 0.0035 & 24.7 & 0.0006 \\
\hline 29.0 & 0.0315 & 46.0 & 0.0089 & 55.1 & 0.0018 \\
\hline 39.8 & 0.0427 & 76.1 & 0.0134 & 85.9 & 0.0030 \\
\hline 51.8 & 0.0684 & 108.7 & 0.0198 & 115.1 & 0.0038 \\
\hline 53.5 & 0.1582 & 160.0 & 0.0322 & 165.0 & 0.0099 \\
\hline 54.0 & 0.2122 & 180.1 & 0.0688 & 230.2 & 0.0125 \\
\hline \multirow[t]{7}{*}{54.3} & 1.0000 & 181.7 & 0.1887 & 290.1 & 0.0255 \\
\hline & & 187.9 & 1.0000 & 354.4 & 0.0285 \\
\hline & & & & 392.0 & 0.0434 \\
\hline & & & & 471.2 & 0.0711 \\
\hline & & & & 490.6 & 0.1739 \\
\hline & & & & 493.1 & 0.2453 \\
\hline & & & & 492.8 & 1.0000 \\
\hline \multicolumn{6}{|c|}{$n$-octane $+\left[4-C_{4} C_{1} p y\right][T C M]$} \\
\hline 1.5 & 0.0026 & 5.8 & 0.0125 & 10.2 & 0.0082 \\
\hline 4.5 & 0.0234 & 14.2 & 0.0307 & 23.4 & 0.0125 \\
\hline 6.6 & 0.0580 & 27.6 & 0.0526 & 46.1 & 0.0198 \\
\hline 6.7 & 0.0914 & 33.0 & 0.0778 & 66.2 & 0.0386 \\
\hline 6.7 & 0.1238 & 33.3 & 0.1322 & 89.8 & 0.0461 \\
\hline 6.7 & 0.1934 & 33.2 & 0.2053 & 103.2 & 0.0600 \\
\hline 6.7 & 0.2565 & 33.3 & 0.2708 & 114.1 & 0.1000 \\
\hline \multirow[t]{3}{*}{6.7} & 1.0000 & 33.4 & 1.0000 & 114.1 & 0.1744 \\
\hline & & & & 114.2 & 0.2851 \\
\hline & & & & 114.2 & 1.0000 \\
\hline
\end{tabular}

${ }^{a}$ Standard uncertainty $(u)$ and relative standard uncertainty $\left(u_{\mathrm{r}}\right)$ are $u(x)=0.0008, u_{\mathrm{r}}(P)=$ 0.02 , and $u(T)=0.1 \mathrm{~K}$ 
Table S2. Continued

\begin{tabular}{|c|c|c|c|c|c|}
\hline$p / \mathrm{kPa}$ & $x_{1}$ & $p / \mathbf{k P a}$ & $x_{1}$ & $p / \mathbf{k P a}$ & $x_{1}$ \\
\hline \multicolumn{2}{|c|}{$\boldsymbol{T} / \mathbf{K}=323$} & \multicolumn{2}{|c|}{$\boldsymbol{T} / \mathbf{K}=363$} & \multicolumn{2}{|c|}{$\boldsymbol{T} / \mathbf{K}=403$} \\
\hline \multicolumn{6}{|c|}{ benzene $+\left[4-C_{4} C_{1} p y\right][T C M]$} \\
\hline 0.6 & 0.0212 & 2.2 & 0.0212 & 6.5 & 0.0147 \\
\hline 1.5 & 0.0541 & 5.2 & 0.0512 & 14.7 & 0.0412 \\
\hline 3.0 & 0.1067 & 10.9 & 0.1010 & 39.5 & 0.0786 \\
\hline 4.5 & 0.1548 & 16.8 & 0.1461 & 54.8 & 0.1126 \\
\hline 6.0 & 0.2028 & 22.9 & 0.1905 & 75.9 & 0.1494 \\
\hline 9.1 & 0.2958 & 36.3 & 0.2741 & 111.5 & 0.2126 \\
\hline 11.3 & 0.3556 & 49.9 & 0.3512 & 164.1 & 0.3013 \\
\hline 15.5 & 0.4395 & 63.0 & 0.4166 & 194.2 & 0.3550 \\
\hline 17.5 & 0.5018 & 73.2 & 0.4665 & 227.2 & 0.4124 \\
\hline 21.0 & 0.5602 & 87.1 & 0.5303 & 254.9 & 0.4648 \\
\hline 24.2 & 0.6094 & 96.9 & 0.5811 & 284.0 & 0.5221 \\
\hline 26.9 & 0.6497 & 105.5 & 0.6293 & 324.9 & 0.6188 \\
\hline 31.2 & 0.7033 & 120.7 & 0.6942 & 340.4 & 0.6635 \\
\hline 34.5 & 0.7361 & 125.2 & 0.7176 & 358.0 & 0.7112 \\
\hline 35.9 & 0.7741 & 130.9 & 0.7565 & 368.4 & 0.7592 \\
\hline 35.9 & 0.8099 & 134.7 & 0.7958 & 378.1 & 0.7973 \\
\hline 36.0 & 0.8302 & 135.9 & 0.8309 & 378.4 & 0.8500 \\
\hline 36.1 & 1.0000 & 136.0 & 1.0000 & 378.4 & 1.0000 \\
\hline \multicolumn{6}{|c|}{$p$-xylene $+\left[4-C_{4} C_{1} p y\right][T C M]$} \\
\hline 0.1 & 0.0201 & 0.8 & 0.0179 & 2.4 & 0.0139 \\
\hline 0.3 & 0.0459 & 1.9 & 0.0425 & 6.2 & 0.0369 \\
\hline 0.6 & 0.0887 & 3.7 & 0.0847 & 12.3 & 0.0737 \\
\hline 0.9 & 0.1284 & 5.4 & 0.1236 & 18.0 & 0.1080 \\
\hline 1.1 & 0.1655 & 6.9 & 0.1620 & 24.2 & 0.1440 \\
\hline 1.7 & 0.2517 & 10.6 & 0.2453 & 34.9 & 0.2164 \\
\hline 2.2 & 0.3271 & 13.5 & 0.3113 & 46.6 & 0.2832 \\
\hline 2.7 & 0.3828 & 16.1 & 0.3670 & 55.7 & 0.3447 \\
\hline 3.1 & 0.4449 & 18.3 & 0.4337 & 61.7 & 0.4051 \\
\hline 3.7 & 0.5001 & 20.4 & 0.4912 & 69.3 & 0.4569 \\
\hline 4.3 & 0.5514 & 22.5 & 0.5430 & 75.5 & 0.5135 \\
\hline 4.3 & 0.6036 & 22.5 & 0.5977 & 79.5 & 0.5670 \\
\hline 4.3 & 0.6467 & 22.6 & 0.6391 & 80.7 & 0.6160 \\
\hline 4.3 & 0.6886 & 22.7 & 0.6841 & 80.8 & 0.6694 \\
\hline 4.3 & 0.7308 & 22.7 & 0.7263 & 80.7 & 0.7108 \\
\hline 4.2 & 0.7690 & 22.8 & 0.7674 & 80.6 & 0.7487 \\
\hline 4.3 & 0.8072 & 22.6 & 0.8063 & 80.6 & 0.7930 \\
\hline 4.3 & 1.0000 & 22.6 & 1.0000 & 80.6 & 1.0000 \\
\hline
\end{tabular}

a Standard uncertainty $(u)$ and relative standard uncertainty $\left(u_{\mathrm{r}}\right)$ are $u(x)=0.0008, u_{\mathrm{r}}(P)=$ 0.02 , and $u(T)=0.1 \mathrm{~K}$ 
Table S3. Vapor-liquid and Vapor-liquid-liquid Equilibria ${ }^{a}$ for $n$-Hexane (1) + Benzene $(2)+\left[C_{2} C_{1}\right.$ im $][$ TCM $](3)$ with $\mathrm{S} / \mathrm{F}=10$

\begin{tabular}{|c|c|c|c|c|c|c|c|c|c|c|c|}
\hline$p / \mathbf{k P a}$ & $y_{1}$ & $y_{2}$ & $x_{1, \mathrm{I}}$ & $x_{2, \mathrm{I}}$ & $x_{1, \mathrm{II}}$ & $x_{2, \mathrm{II}}$ & $x_{3, \mathrm{II}}$ & $x_{1}$ & $x_{2}$ & $x_{3}$ & $\alpha_{21}$ \\
\hline \multicolumn{12}{|c|}{$T / \mathrm{K}=323.2$} \\
\hline 8.9 & 0.0000 & 1.0000 & & & & & & 0.0000 & 0.1963 & 0.8037 & \\
\hline 26.2 & 0.6889 & 0.3111 & & & & & & 0.0060 & 0.1775 & 0.8164 & 65.3 \\
\hline 40.9 & 0.8207 & 0.1793 & & & & & & 0.0142 & 0.1572 & 0.8286 & 50.7 \\
\hline 51.0 & 0.8742 & 0.1258 & & & & & & 0.0239 & 0.1414 & 0.8347 & 41.0 \\
\hline 53.4 & 0.9032 & 0.0968 & 0.8412 & 0.1588 & 0.0160 & 0.1171 & 0.8669 & 0.0380 & 0.1182 & 0.8438 & 29.0 \\
\hline 53.5 & 0.9205 & 0.0795 & 0.8766 & 0.1234 & 0.0171 & 0.0943 & 0.8886 & 0.0561 & 0.0956 & 0.8483 & 19.7 \\
\hline 53.6 & 0.9348 & 0.0652 & 0.8982 & 0.1018 & 0.0175 & 0.0755 & 0.9070 & 0.0747 & 0.0772 & 0.8481 & 14.8 \\
\hline 53.7 & 0.9513 & 0.0487 & 0.9260 & 0.0740 & 0.0171 & 0.0560 & 0.9269 & 0.0962 & 0.0575 & 0.8463 & 11.7 \\
\hline 53.8 & 0.9686 & 0.0314 & 0.9532 & 0.0468 & 0.0170 & 0.0362 & 0.9468 & 0.1134 & 0.0373 & 0.8493 & 10.1 \\
\hline 53.9 & 0.9823 & 0.0177 & 0.9741 & 0.0259 & 0.0163 & 0.0205 & 0.9632 & 0.1308 & 0.0211 & 0.8481 & 9.0 \\
\hline 54.4 & 1.0000 & 0.0000 & 1.0000 & 0.0000 & 0.0171 & 0.0000 & 0.9829 & 0.1489 & 0.0000 & 0.8511 & \\
\hline \multicolumn{12}{|c|}{$T / \mathrm{K}=363.2$} \\
\hline 47.2 & 0.0000 & 1.0000 & & & & & & 0.0000 & 0.1745 & 0.8255 & \\
\hline 71.5 & 0.5059 & 0.4941 & & & & & & 0.0020 & 0.1653 & 0.8327 & 84.6 \\
\hline 86.9 & 0.6263 & 0.3737 & & & & & & 0.0029 & 0.1437 & 0.8533 & 82.1 \\
\hline 106.1 & 0.6925 & 0.3075 & & & & & & 0.0036 & 0.1264 & 0.8700 & 78.9 \\
\hline 130.7 & 0.7936 & 0.2064 & & & & & & 0.0060 & 0.1094 & 0.8867 & 70.1 \\
\hline 149.4 & 0.8765 & 0.1235 & & & & & & 0.0111 & 0.0922 & 0.8967 & 58.9 \\
\hline 161.5 & 0.9144 & 0.0856 & & & & & & 0.0224 & 0.0772 & 0.9004 & 36.8 \\
\hline 187.5 & 0.9466 & 0.0534 & 0.9293 & 0.0707 & 0.0157 & 0.0601 & 0.9242 & 0.0289 & 0.0609 & 0.9103 & 37.4 \\
\hline 188.4 & 0.9642 & 0.0358 & 0.9531 & 0.0469 & 0.0156 & 0.0393 & 0.9451 & 0.0441 & 0.0400 & 0.9159 & 24.4 \\
\hline 188.1 & 0.9847 & 0.0153 & 0.9848 & 0.0152 & 0.0158 & 0.0126 & 0.9716 & 0.0670 & 0.0190 & 0.9141 & 18.2 \\
\hline 188.1 & 1.0000 & 0.0000 & 0.9995 & 0.0005 & 0.0167 & 0.0000 & 0.9833 & 0.0606 & 0.0000 & 0.9394 & \\
\hline
\end{tabular}


Table S3. Continued

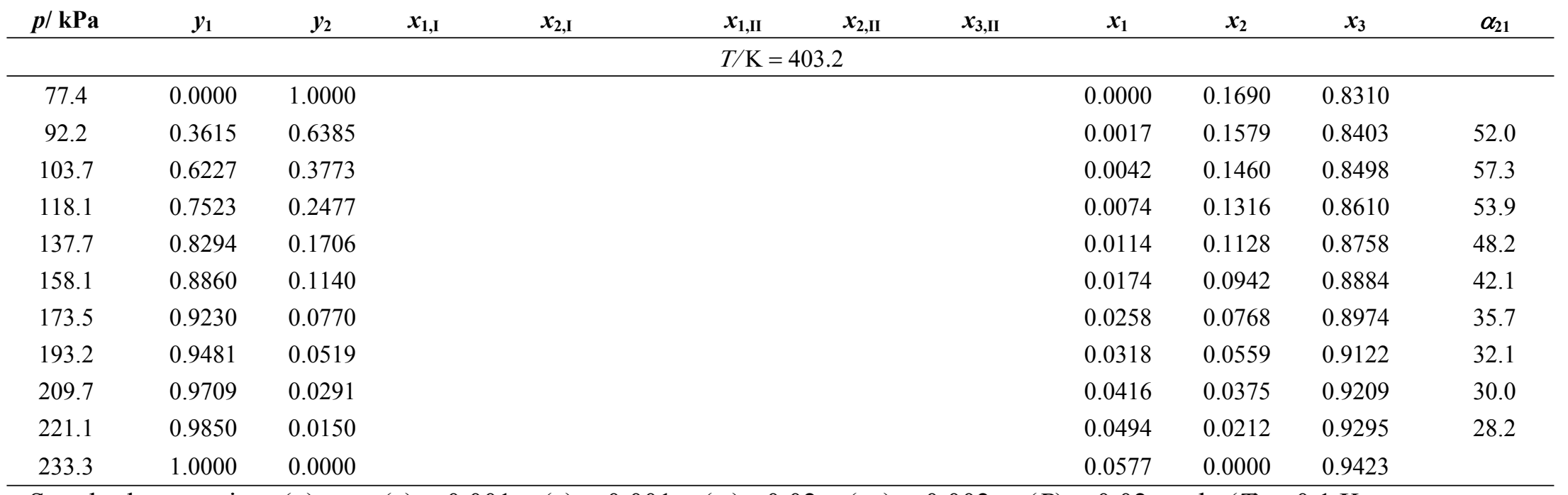

a Standard uncertainty $(u)$ are $u(y)=0.001, u(x)=0.001, u\left(x_{\mathrm{I}}\right)=0.02, u\left(x_{\mathrm{II}}\right)=0.002, u_{\mathrm{r}}(P)=0.02$, and $u(T)=0.1 \mathrm{~K}$. 
Table S4. Vapor-liquid and Vapor-liquid-liquid Equilibria ${ }^{a}$ for $n$-Hexane (1) + Benzene (2) $+\left[4-C_{4} C_{1}\right.$ py $][T C M](3)$ with $S / F=10$

\begin{tabular}{|c|c|c|c|c|c|c|c|c|c|c|c|}
\hline$p / \mathbf{k P a}$ & $y_{1}$ & $y_{2}$ & $x_{1, \mathrm{I}}$ & $x_{2, \mathrm{I}}$ & $x_{1, \mathrm{II}}$ & $x_{2, \mathrm{II}}$ & $x_{3, \mathrm{II}}$ & $x_{1}$ & $x_{2}$ & $x_{3}$ & $\alpha_{21}$ \\
\hline \multicolumn{12}{|c|}{$T / \mathrm{K}=323.2$} \\
\hline 9.1 & 0.0000 & 1.0000 & & & & & & 0.0000 & 0.2319 & 0.7681 & \\
\hline 17.0 & 0.6859 & 0.3141 & & & & & & 0.0125 & 0.2117 & 0.7758 & 37.0 \\
\hline 28.0 & 0.8382 & 0.1618 & & & & & & 0.0237 & 0.1823 & 0.7939 & 39.8 \\
\hline 40.9 & 0.8877 & 0.1123 & & & & & & 0.0324 & 0.1626 & 0.8050 & 39.7 \\
\hline 51.7 & 0.9270 & 0.0730 & & & & & & 0.0444 & 0.1395 & 0.8161 & 39.9 \\
\hline 53.6 & 0.9427 & 0.0573 & 0.8852 & 0.1148 & 0.0312 & 0.1140 & 0.8548 & 0.0652 & 0.1141 & 0.8207 & 28.8 \\
\hline 53.7 & 0.9526 & 0.0474 & 0.9049 & 0.0951 & 0.0304 & 0.0921 & 0.8775 & 0.0880 & 0.0923 & 0.8198 & 21.1 \\
\hline 53.9 & 0.9662 & 0.0338 & 0.9333 & 0.0667 & 0.0300 & 0.0669 & 0.9031 & 0.1085 & 0.0669 & 0.8245 & 17.6 \\
\hline 54.0 & 0.9773 & 0.0227 & 0.9580 & 0.0420 & 0.0306 & 0.0440 & 0.9255 & 0.1303 & 0.0437 & 0.8260 & 14.5 \\
\hline 54.0 & 0.9881 & 0.0119 & 0.9773 & 0.0227 & 0.0313 & 0.0242 & 0.9445 & 0.1441 & 0.0240 & 0.8319 & 13.8 \\
\hline 54.4 & 1.0000 & 0.0000 & 1.0000 & 0.0000 & 0.0314 & 0.0000 & 0.9686 & 0.1609 & 0.0000 & 0.8391 & \\
\hline \multicolumn{12}{|c|}{$T / \mathrm{K}=363.2$} \\
\hline 25.8 & 0.0000 & 1.0000 & & & & & & 0.0000 & 0.2075 & 0.7925 & \\
\hline 45.5 & 0.5140 & 0.4860 & & & & & & 0.0038 & 0.1915 & 0.8047 & 53.3 \\
\hline 67.7 & 0.6460 & 0.3540 & & & & & & 0.0066 & 0.1892 & 0.8222 & 52.1 \\
\hline 89.0 & 0.7589 & 0.2411 & & & & & & 0.0091 & 0.1495 & 0.8414 & 51.9 \\
\hline 111.3 & 0.8283 & 0.1717 & & & & & & 0.0114 & 0.1289 & 0.8597 & 54.7 \\
\hline 138.4 & 0.8924 & 0.1076 & & & & & & 0.0178 & 0.1120 & 0.8702 & 52.2 \\
\hline 152.6 & 0.9275 & 0.0725 & & & & & & 0.0215 & 0.0888 & 0.8898 & 52.9 \\
\hline 174.9 & 0.9533 & 0.0467 & & & & & & 0.0289 & 0.0689 & 0.9021 & 48.6 \\
\hline 187.2 & 0.9726 & 0.0274 & 0.9730 & 0.0270 & 0.0274 & 0.0470 & 0.9256 & 0.0426 & 0.0474 & 0.9101 & 39.5 \\
\hline 187.5 & 0.9886 & 0.0114 & 0.9886 & 0.0114 & 0.0282 & 0.0203 & 0.9515 & 0.0489 & 0.0205 & 0.9307 & 36.3 \\
\hline 188.1 & 1.0000 & 0.0000 & 0.9995 & 0.0005 & 0.0286 & 0.0000 & 0.9714 & 0.0638 & 0.0000 & 0.9362 & \\
\hline
\end{tabular}


Table S4. Continued

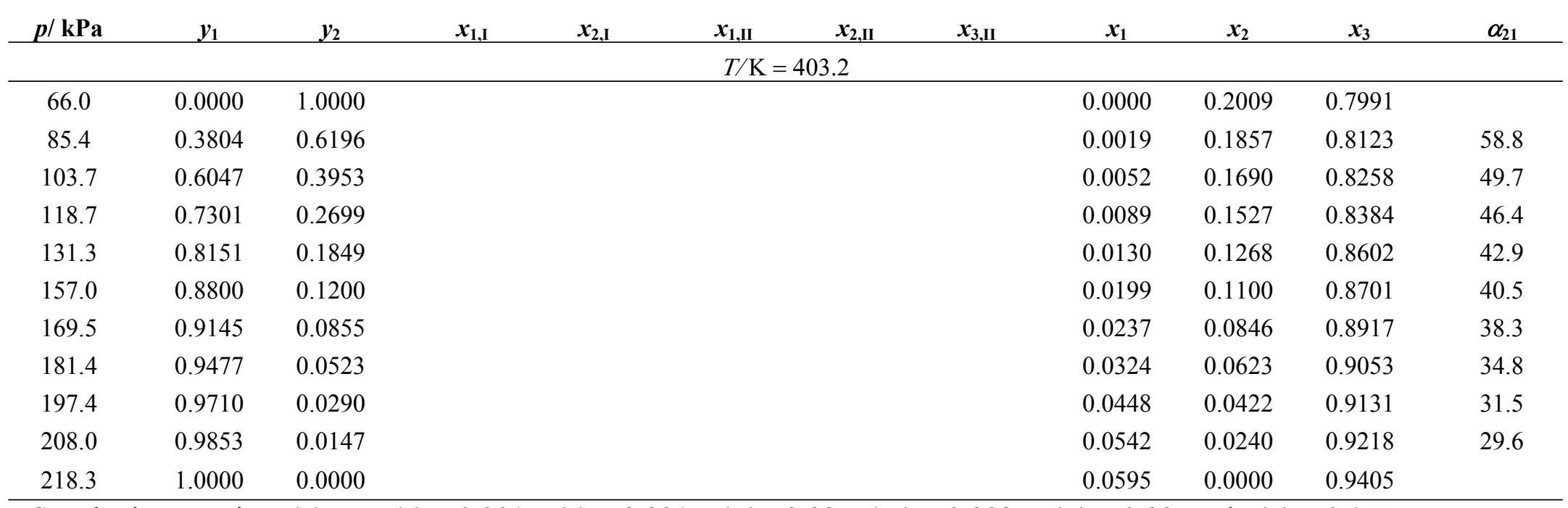

a Standard uncertainty $(u)$ are $u(y)=0.001, u(x)=0.001, u\left(x_{\mathrm{I}}\right)=0.02, u\left(x_{\mathrm{II}}\right)=0.002, u_{\mathrm{r}}(P)=0.02$, and $u(T)=0.1 \mathrm{~K}$ 
Table S5. Vapor-liquid and Vapor-liquid-liquid Equilibria ${ }^{a}$ for $n$-Octane (1) + -Xylene $(2)+\left[\mathrm{C}_{2} \mathrm{C}_{1}\right.$ im $][\mathrm{TCM}](3)$ with $\mathrm{S} / \mathrm{F}=10$

\begin{tabular}{|c|c|c|c|c|c|c|c|c|c|c|c|}
\hline$p / \mathbf{k P a}$ & $y_{1}$ & $y_{2}$ & $x_{1, \mathrm{I}}$ & $x_{2, \mathrm{I}}$ & $x_{1, \mathrm{II}}$ & $x_{2, \mathrm{II}}$ & $x_{3, \mathrm{II}}$ & $x_{1}$ & $x_{2}$ & $x_{3}$ & $\alpha_{21}$ \\
\hline \multicolumn{12}{|c|}{$T / \mathrm{K}=323.2$} \\
\hline 1.9 & 0.0000 & 1.0000 & & & & & & 0.0000 & 0.1598 & 0.8402 & \\
\hline 5.6 & 0.6225 & 0.3775 & 0.5182 & 0.4818 & 0.0043 & 0.1431 & 0.8526 & 0.0131 & 0.1492 & 0.8377 & 18.8 \\
\hline 5.9 & 0.6806 & 0.3194 & 0.5793 & 0.4207 & 0.0044 & 0.1207 & 0.8749 & 0.0286 & 0.1336 & 0.8378 & 9.9 \\
\hline 6.0 & 0.7538 & 0.2462 & 0.6606 & 0.3394 & 0.0050 & 0.0974 & 0.8976 & 0.0436 & 0.1119 & 0.8445 & 7.9 \\
\hline 6.2 & 0.7910 & 0.2090 & 0.7155 & 0.2845 & 0.0055 & 0.0821 & 0.9124 & 0.0579 & 0.0972 & 0.8449 & 6.4 \\
\hline 6.3 & 0.8295 & 0.1705 & 0.7739 & 0.2261 & 0.0059 & 0.0667 & 0.9274 & 0.0721 & 0.0807 & 0.8472 & 5.4 \\
\hline 6.5 & 0.8681 & 0.1319 & 0.8232 & 0.1768 & 0.0059 & 0.0525 & 0.9416 & 0.0874 & 0.0650 & 0.8475 & 4.9 \\
\hline 6.4 & 0.9015 & 0.0985 & 0.8764 & 0.1236 & 0.0063 & 0.0393 & 0.9544 & 0.1026 & 0.0487 & 0.8487 & 4.3 \\
\hline 6.6 & 0.9368 & 0.0632 & 0.9195 & 0.0805 & 0.0062 & 0.0251 & 0.9687 & 0.1175 & 0.0319 & 0.8506 & 4.0 \\
\hline 6.7 & 0.9679 & 0.0321 & 0.9625 & 0.0375 & 0.0068 & 0.0128 & 0.9804 & 0.1326 & 0.0160 & 0.8514 & 3.6 \\
\hline 6.7 & 1.0000 & 0.0000 & 1.0000 & 0.0000 & 0.0069 & 0.0000 & 0.9931 & 0.1465 & 0.0000 & 0.8535 & \\
\hline \multicolumn{12}{|c|}{$T / \mathrm{K}=363.2$} \\
\hline 12.6 & 0.0000 & 1.0000 & & & & & & 0.0000 & 0.1749 & 0.8251 & \\
\hline 27.2 & 0.5639 & 0.4361 & & & & & & 0.0081 & 0.1605 & 0.8314 & 25.7 \\
\hline 30.2 & 0.6869 & 0.3131 & 0.5579 & 0.4421 & 0.0055 & 0.1315 & 0.8630 & 0.0214 & 0.1405 & 0.8380 & 14.4 \\
\hline 31.0 & 0.7363 & 0.2637 & 0.6307 & 0.3693 & 0.0061 & 0.1101 & 0.8838 & 0.0392 & 0.1239 & 0.8369 & 8.8 \\
\hline 31.6 & 0.7959 & 0.2041 & 0.6979 & 0.3021 & 0.0067 & 0.0923 & 0.9010 & 0.0533 & 0.1065 & 0.8401 & 7.8 \\
\hline 31.9 & 0.8145 & 0.1855 & 0.7502 & 0.2498 & 0.0070 & 0.0748 & 0.9182 & 0.0705 & 0.0898 & 0.8397 & 5.6 \\
\hline 32.1 & 0.8554 & 0.1446 & 0.7994 & 0.2006 & 0.0068 & 0.0572 & 0.9360 & 0.0859 & 0.0715 & 0.8426 & 4.9 \\
\hline 32.3 & 0.8945 & 0.1055 & 0.8560 & 0.1440 & 0.0069 & 0.0418 & 0.9513 & 0.1013 & 0.0532 & 0.8454 & 4.5 \\
\hline 32.8 & 0.9335 & 0.0665 & 0.9078 & 0.0922 & 0.0075 & 0.0273 & 0.9652 & 0.1185 & 0.0353 & 0.8462 & 4.2 \\
\hline 33.1 & 0.9666 & 0.0334 & 0.9540 & 0.0460 & 0.0074 & 0.0134 & 0.9792 & 0.1356 & 0.0178 & 0.8466 & 3.8 \\
\hline 33.4 & 1.0000 & 0.0000 & 1.0000 & 0.0000 & 0.0077 & 0.0000 & 0.9923 & 0.1506 & 0.0000 & 0.8494 & \\
\hline
\end{tabular}

${ }^{\text {a }}$ Standard uncertainty $(u)$ are $u(y)=0.001, u(x)=0.001, u\left(x_{\mathrm{I}}\right)=0.02, u\left(x_{\mathrm{II}}\right)=0.002, u_{\mathrm{r}}(P)=0.02$, and $u(T)=0.1 \mathrm{~K}$. 
Table S5. Continued

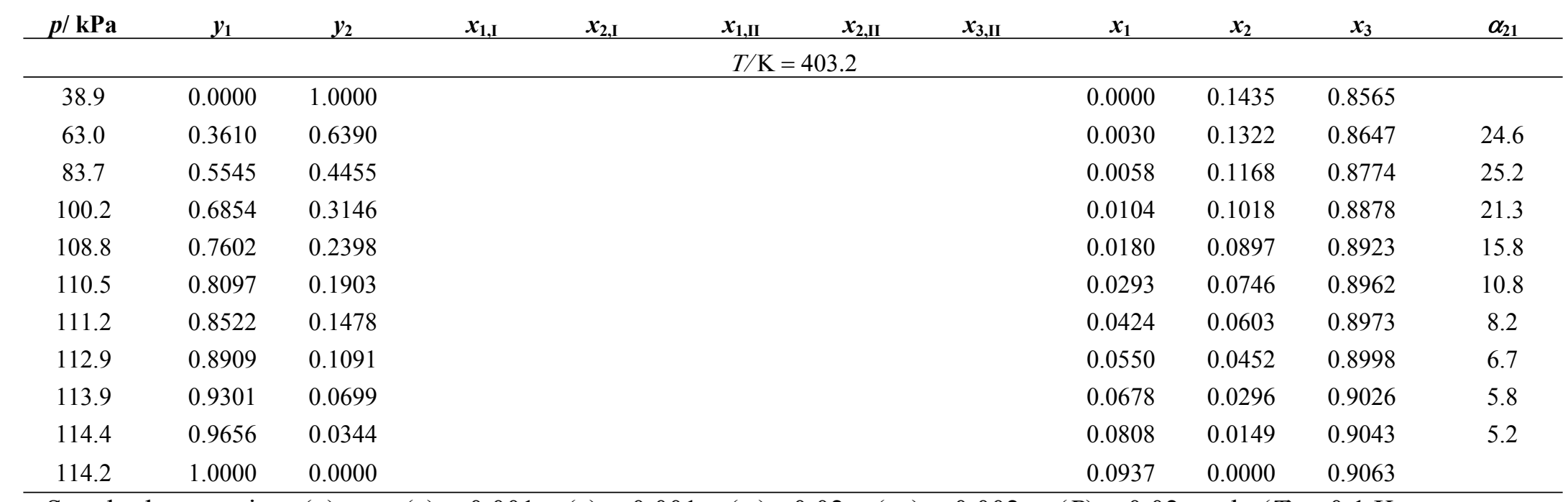

a Standard uncertainty $(u)$ are $u(y)=0.001, u(x)=0.001, u\left(x_{\mathrm{I}}\right)=0.02, u\left(x_{\mathrm{II}}\right)=0.002, u_{\mathrm{r}}(P)=0.02$, and $u(T)=0.1 \mathrm{~K}$. 
Table S6. Vapor-liquid and Vapor-liquid-liquid Equilibria ${ }^{a}$ for $n$-Octane (1) + -Xylene $(2)+\left[4-C_{4} C_{1}\right.$ py $][T C M](3)$ with $S / F=10$

\begin{tabular}{|c|c|c|c|c|c|c|c|c|c|c|c|}
\hline$p / \mathbf{k P a}$ & $y_{1}$ & $y_{2}$ & $x_{1, \mathrm{I}}$ & $x_{2, \mathrm{I}}$ & $x_{1, \mathrm{II}}$ & $x_{2, \mathrm{II}}$ & $x_{3, \mathrm{II}}$ & $x_{1}$ & $x_{2}$ & $x_{3}$ & $\alpha_{21}$ \\
\hline \multicolumn{12}{|c|}{$T / \mathrm{K}=323.2$} \\
\hline 1.0 & 0.0000 & 1.0000 & & & & & & 0.0000 & 0.1855 & 0.8145 & \\
\hline 4.5 & 0.7034 & 0.2966 & & & & & & 0.0147 & 0.1709 & 0.8144 & 27.5 \\
\hline 6.4 & 0.8260 & 0.1740 & 0.6238 & 0.3762 & 0.0080 & 0.1420 & 0.8500 & 0.0308 & 0.1510 & 0.8182 & 23.3 \\
\hline 6.5 & 0.8537 & 0.1463 & 0.6946 & 0.3054 & 0.0087 & 0.1195 & 0.8718 & 0.0496 & 0.1308 & 0.8196 & 15.4 \\
\hline 6.6 & 0.8653 & 0.1347 & 0.7497 & 0.2503 & 0.0092 & 0.1009 & 0.8899 & 0.0658 & 0.1125 & 0.8217 & 11.0 \\
\hline 6.7 & 0.8937 & 0.1063 & 0.7977 & 0.2023 & 0.0099 & 0.0836 & 0.9066 & 0.0836 & 0.0948 & 0.8216 & 9.5 \\
\hline 6.6 & 0.9182 & 0.0818 & 0.8428 & 0.1572 & 0.0104 & 0.0654 & 0.9242 & 0.1003 & 0.0755 & 0.8242 & 8.4 \\
\hline 6.7 & 0.9395 & 0.0605 & 0.8848 & 0.1152 & 0.0108 & 0.0484 & 0.9408 & 0.1182 & 0.0567 & 0.8251 & 7.4 \\
\hline 6.7 & 0.9603 & 0.0397 & 0.9242 & 0.0758 & 0.0104 & 0.0309 & 0.9587 & 0.1355 & 0.0371 & 0.8273 & 6.6 \\
\hline 6.7 & 0.9796 & 0.0204 & 0.9626 & 0.0374 & 0.0105 & 0.0154 & 0.9740 & 0.1540 & 0.0188 & 0.8273 & 5.9 \\
\hline 6.7 & 1.0000 & 0.0000 & 1.0000 & 0.0000 & 0.0107 & 0.0000 & 0.9894 & 0.1696 & 0.0000 & 0.8304 & \\
\hline \multicolumn{12}{|c|}{$T / \mathrm{K}=363.2$} \\
\hline 8.1 & 0.0000 & 1.0000 & & & & & & 0.0000 & 0.2044 & 0.7956 & \\
\hline 17.5 & 0.5856 & 0.4144 & & & & & & 0.0122 & 0.1864 & 0.8013 & 21.5 \\
\hline 27.3 & 0.7644 & 0.2356 & & & & & & 0.0247 & 0.1666 & 0.8086 & 21.9 \\
\hline 31.9 & 0.8341 & 0.1659 & 0.6787 & 0.3213 & 0.0087 & 0.1360 & 0.8553 & 0.0422 & 0.1453 & 0.8125 & 17.3 \\
\hline 32.8 & 0.8712 & 0.1288 & 0.7316 & 0.2684 & 0.0091 & 0.1142 & 0.8767 & 0.0587 & 0.1249 & 0.8164 & 14.4 \\
\hline 32.6 & 0.8822 & 0.1178 & 0.7848 & 0.2152 & 0.0097 & 0.0942 & 0.8961 & 0.0787 & 0.1050 & 0.8163 & 10.0 \\
\hline 32.7 & 0.9117 & 0.0883 & 0.8295 & 0.1705 & 0.0100 & 0.0738 & 0.9162 & 0.0973 & 0.0841 & 0.8186 & 8.9 \\
\hline 32.9 & 0.9265 & 0.0735 & 0.8726 & 0.1274 & 0.0101 & 0.0538 & 0.9362 & 0.1169 & 0.0629 & 0.8201 & 6.8 \\
\hline 33.3 & 0.9591 & 0.0409 & 0.9180 & 0.0820 & 0.0108 & 0.0351 & 0.9541 & 0.1358 & 0.0415 & 0.8227 & 7.2 \\
\hline 33.3 & 0.9778 & 0.0222 & 0.9611 & 0.0389 & 0.0105 & 0.0172 & 0.9722 & 0.1518 & 0.0204 & 0.8278 & 5.9 \\
\hline 33.4 & 1.0000 & 0.0000 & 1.0000 & 0.0000 & 0.0107 & 0.0000 & 0.9893 & 0.1747 & 0.0000 & 0.8253 & \\
\hline
\end{tabular}

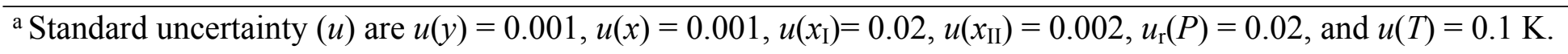


Table S6. Continued

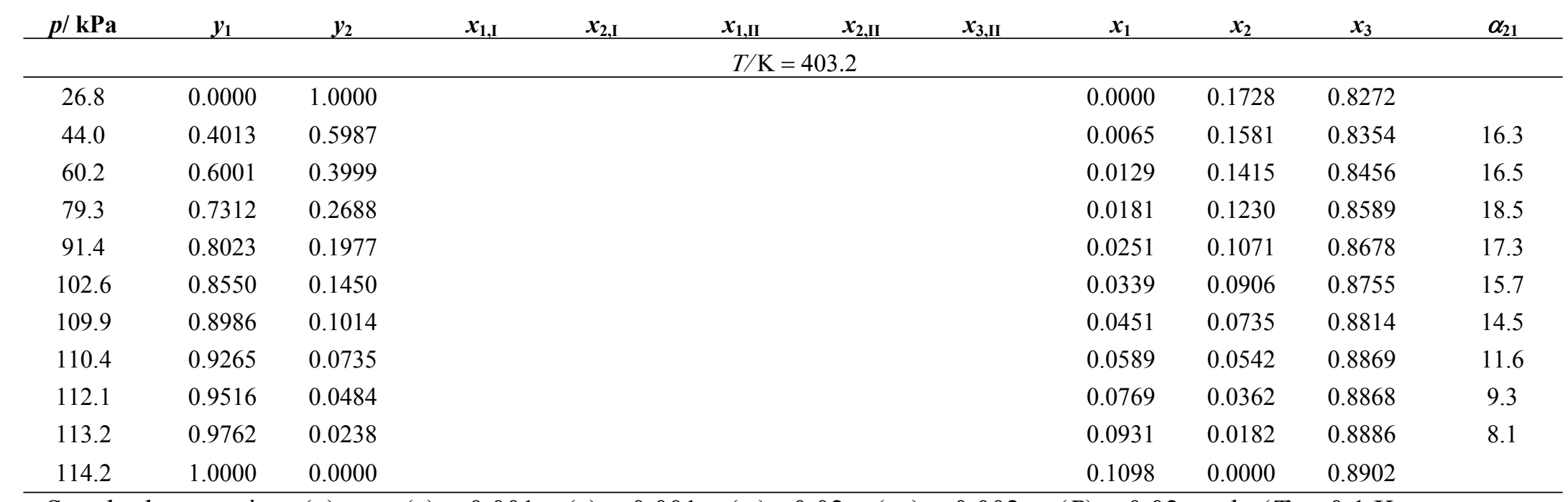

a Standard uncertainty $(u)$ are $u(y)=0.001, u(x)=0.001, u\left(x_{\mathrm{I}}\right)=0.02, u\left(x_{\mathrm{II}}\right)=0.002, u_{\mathrm{r}}(P)=0.02$, and $u(T)=0.1 \mathrm{~K}$. 